\title{
Review: epidural or spinal anaesthesia reduces post-operative mortality and morbidity
}

\author{
Rodgers A, Walker N, Schug S, et al. Reduction of postoperative mortality and morbidity with epidural or spinal \\ anaesthesia: results from overview of randomised trials. BMJ 2000 Dec 16;321:1493-7.

\section{QUESTION: What is the effect of epidural or spinal anaesthesia on post-operative mortality and morbidity?}

\section{Data sources}

Studies were identified by searching Medline (1966-96), Current Contents (1995-6), EMBASE/Excerpta Medica (1980-96), and the Cochrane Library (1998) with terms that include regional anaesthesia, spinal, and epidural. In addition, conference proceedings were hand searched, bibliographies of relevant papers were scanned, and authors were contacted.

\section{Study selection}

Studies were selected if they were randomised trials of epidural or spinal anaesthesia compared with general anaesthesia and if data were available before January 1 , 1997.

\section{Data extraction}

Data were extracted on study characteristics, including type of surgery, patient numbers, type of neuraxial blockade used, and outcomes.

\section{Main results}

158 studies were identified, and 141 studies (9559 patients, 4871 who received epidural or spinal anaesthesia and 4688 who did not) met the selection criteria. Studies represented various surgical groups: general surgery $(n=28)$, orthopaedics $(n=44)$, urology $(n=18)$, vascular $(n=22)$, and other $(n=29) .247$ deaths that occurred within 30 days of randomisation were reported in 35 trials. Epidural or spinal anaesthesia reduced overall 30 day mortality, deep venous thrombosis, pulmonary embolism, perioperative and post-operative bleeding requiring transfusion, and pneumonia (table). No difference existed for the effect of epidural or spinal anaesthesia on mortality among different surgical groups, between spinal and epidural anaesthesia on

Neuraxial blockade $v$ no neuraxial blockade on mortality and morbidity at 30 days*

\begin{tabular}{|c|c|c|c|c|}
\hline \multirow[b]{2}{*}{ Outcomes } & \multicolumn{2}{|c|}{ Weighted event rates } & \multirow[b]{2}{*}{ RRR $(95 \% \mathrm{Cl})$} & \multirow[b]{2}{*}{ NNT (Cl) } \\
\hline & Blockade & No blockade & & \\
\hline Mortality & $2 \%$ & $3 \%$ & $31 \%(12$ to 46$)$ & 104 (62 to 310$)$ \\
\hline Deep venous thrombosis & $3 \%$ & $5 \%$ & $37 \%$ (22 to 48$)$ & 58 (40 to 105$)$ \\
\hline Pulmonary embolism & $0.6 \%$ & $1.4 \%$ & $56 \%$ (33 to 71$)$ & $126(82$ to 251$)$ \\
\hline Perioperative transfusion & $4 \%$ & $6 \%$ & $34 \%$ (21 to 45$)$ & 50 (35 to 87 ) \\
\hline Post-operative transfusion & $0.6 \%$ & $1.5 \%$ & $57 \%$ (34 to 72$)$ & 120 (79 to 230$)$ \\
\hline Pneumonia & $3 \%$ & $5 \%$ & $40 \%(26$ to 51$)$ & 50 (35 to 81$)$ \\
\hline
\end{tabular}

*Abbreviations defined in glossary; RRR, NNT, and $\mathrm{Cl}$ calculated from data in article. mortality, or between trials of spinal or epidural anaesthesia plus general anaesthesia and trials of spinal or epidural anaesthesia alone.

\section{Conclusion}

Epidural or spinal anaesthesia reduces post-operative mortality, deep venous thrombosis, pulmonary embolism, bleeding, and pneumonia.

\section{COMMENTARY}

The systematic review by Rodgers et al provides evidence from randomised trials that regional anaesthesia is associated with reduced mortality and morbidity in patients having surgery. The study was well done, and the authors tried to evaluate the potential of bias on the results.

None the less, when evaluating the results, several points should be considered. Firstly, the test of heterogeneity was not statistically significant, which shows that the results did not differ systematically among subgroups. However, the 95\% confidence interval for the effect of neuraxial blockade on mortality, although favouring neuraxial blockade, crossed the value of 1 for all surgical types except orthopaedic patients. Whether these subgroup results are caused by insufficient power from a limited sample size or by a true lack of treatment effect is unclear. Secondly, of the included studies, 87 of $107(81 \%)$ had $\leqslant 50$ patients, which raises concerns about study quality. Thirdly, 51 of 107 studies (48\%) were published before 1986, and 99 of 107 studies (93\%) were published before 1991, when mortality from general anaesthesia was higher and prophylaxis for deep venous thrombosis was infrequently used. How these temporal changes may influence the findings is unclear.

How then do we advise patients about which type of anaesthesia to use? As always, we must balance the risks and benefits. This review provides evidence for improved survival and reduced complications in patients receiving neuraxial blockade without evidence of harm. Anaesthesiologists should discuss these findings with patients when considering the type of anaesthesia. Further research is needed to evaluate whether these findings hold true with current anaesthetic practice and to provide more precise estimates of the effect of neuraxial blockade in different types of surgery. Meanwhile, the evidence supports the use of neuraxial blockade when possible.

Peter J Pronovost, $\mathrm{MD}, \mathrm{PhD}$ Johns Hopkins University Baltimore, Maryland, USA 